\title{
Article
}

\section{Determination of the Carnitine and Acylcarnitine Profile in Patients with Lipoid Proteinosis}

\author{
Ataman Gönel 1,*, Ismail Koyuncu ${ }^{2}$, Mustafa Aksoy ${ }^{3}$ and Hakim Çelik ${ }^{4}$ \\ 1 Department of Medical Biochemistry, Harran University, Turkey; atamangonel@gmail.com \\ 2 Department of Medical Biochemistry, Harran University, Turkey; ismailkoyuncu1@gmail.com \\ 3 Department of Dermatology Harran University, Turkey; derma63@gmail.com \\ 4 Department of Medical Physiology, Harran University, Turkey; hakimcell@gmail.com \\ * Correspondence: atamangonel@gmail.com; Tel.: +90-507-5669870
}

\begin{abstract}
Background and objectives: Lipoid proteinosis (LP) is an autosomal recessive transfer lysosomal storage disease, characterised by the accumulation of hyalin substance in the mucous membranes, skin, internal organs and brain, for which there is no biochemical diagnostic method. The aim of this study was to determine the carnitine and acylcarnitine metabolic profile with LC-MS/MS in LP patients and thereby examine the potential of this as a new biochemical method in the determination of biochemical markers in LP patients. Materials and Methods: In this study, 27 carnitine and acylcarnitine esters were measured with LC-MS/MS in serum samples taken from 14 healthy control subjects and 14 patients who presented at the Skin and Venereal Diseases Polyclinic and were diagnosed with LP as a result of clinical, radiological and histopathological examinations. Results: The results of the study showed that C0 (free carnitine) C3, C4, C4:DC, C5DC, C6, C8, C14:1, C14:2, C16 and C18 acylcarnitines were statistically significantly reduced in the LP patients $(\mathrm{p}<0.05, \mathrm{p}<0.01)$. Conclusions: It was concluded that the application of carnitine profile screening, which is an inexpensive, rapid and reliable method, could make a contribution to the differential diagnosis as aa supporting laboratory test in individuals with suspected LP.
\end{abstract}

Keywords: LC-MS/MS; Lipoid proteinosis; Carnitine; Acylcarnitine

\section{Introduction}

Lipoid proteinosis (LP), which is known as hyalinosis cutis et mucosae or Urbach-Wiethe disease, is an infrequently seen lysosomal storage disease with a chronic course that is characterised by hyalin substance accumulation in mucous membranes, skin, internal organs and the brain [1]. The clinical findings of LP show variations and generally start with voice restrictions in the neonatal period. Various skin symptoms emerge in childhood such as papillary, acneform scars at the eyelash roots and wart-like papillae and plaque. A wide clinical heterogenity may be seen even within a family or an isolated patient population [2]. In a 2002 study using genome link analysis, pathogen mutations of ECM1 gene with an accompanying location on chromosome 1q21 were identified in LP [3]. Since that time a total of 52 different pathogen mutations have been reported, including missense, small and large delesions and insertions. Approximately $50 \%$ of the mutations have been shown to be in exons 6 and 7 [2].

Although some data has been obtained about genetic mutations in LP disease, there is insufficient information about the biochemical pathophysiology. Knowing which metabolic pathways are cut associated with genetic mutations could be useful in respect of disease prognosis and management of complications. LC-MS/MS devices, which have recently become widespread in the assignment of biochemical metabolites, could be of guidance in the determination of pathological pathways. LC/MS-MS reflects processed information of the levels of metabolites of cellular functions and thus identifies phenotypes of tissues or cells associated with genetic or environmental changes [4-6]. The normal acylcarnitine balance in healthy individuals changes because of metabolic changes. The determination of free carnitine and acylcarnitine profiles using 
mass spectrometry measurement methods provides a significant contribution about the pathogenesis of diseases [7,8].

The aim of the current study was to determine the carnitine and acylcarnitine metabolic profiles with LC-MS/MS in LP patients and to establish that this could be a new method in the objective determination of biochemical markers in LP patients.

\section{Materials and Methods}

\subsection{Clinical Samples:}

The study included 14 patients who presented at the Skin and Venereal Diseases Polyclinic and were diagnosed with LP as a result of clinical, radiological and histopathological examinations and a control group of 14 age and gender-matched healthy individuals. Patients were excluded from the study if they were pregnant or breastfeeding, smoked cigarettes or drank alcohol, were uncooperative or had a systemic disease. Approval for the study was granted by the Local Ethics Committee (Harran University, Medical Faculty, number: 05-07-23, date: 01.02.2018) and the study was designed and conducted in accordance with the principles of the Helsinki Declaration. Informed consent was obtained from all the study participants. Blood samples were taken in the morning after 8 hours of fasting. The samples were withdrawn into gel tubes, centrifuged at $5000 \mathrm{rpm}$ for $10 \mathrm{mins}$, then the separated serums were placed in Eppendorf tubes and stored at $-80^{\circ} \mathrm{C}$ until assay. A total of 27 parameters of free carnitine and acylcarnitine esters were measured (C0, C2, C3, C4, C4DC, C5, C5:1, C5OH, C5DC, C6, C6DC, C8, C8:1, C8DC, C10, C10:1, C10DC, C12, C14, C14:1, C14:2, C16, C16:1, C18, C18:1, C18:2, C18:1 OH). The acylcarnitine esters were measured using a Shimadzu Nexera X2 ultra high performance liquid chromatograph (UHPLC) coupled with a Shimadzu 8040 triple quadrupole mass spectrometer (MS/MS) device (Shimadzu North America, Columbia, MD, USA).

2.2. Materials and Reactives: HPLC graded formic acid, methanol, Acenonitrile Sigma (Loughborough, UK) acylcarnitine internal standard (Cambridge isotop lab) were purchased.

2.3. Sample preparation: The sample preparation for routine screening tests was applied exactly as reported by la Marca et al in 2008, with the exception of the butylation procedure, which was not conducted. The carnitine profile was examined with a modification to the neonatal screening method developed by LaMarca and Azzari [9,10]. Filter paper (Whatman filter paper 10538018) was cut to discs of $3.2 \mathrm{~mm}$ diameter and placed in 96 -well plates. Then $5 \mu \mathrm{l}$ plasma sample was placed in each well and left to dry at room temperature overnight.

2.4. Tandem mass spectrometry: The sample was extracted by dispensing $300 \mu \mathrm{L}$ of an extraction solution consisting of a mixture of methanol and aqueous solution of $3 \mathrm{mmol} / \mathrm{L}$ hydrate hydrazine at an approximate relative volume/volume ratio of $66.6 \%$ and $33.3 \%$, respectively. Internal standards, stable heavy isotope analogues of carnitine and acylcarnitines, were also present in the extract solution. The extracted sample was injected into Shimadzu LCMS-8040. Mass spectral data for the acylcarnitines were acquired through a precursor ion scan of $85 \mathrm{~m} / \mathrm{z}$ in positive mode (CE-25V). The percentage of each analyte recovered was determined through comparison with an internal standard for each analyte. The Standard Concentrations were in the range of 7.6-152 $\mu \mathrm{mol} / \mathrm{L}$ for acylcarnitines. Spiked samples with different concentrations of analyte were used as daily control quality tests.

2.5. Analysis Condition: A run of 2.2 minutes was applied in FIA Flow $0.070 \mu \mathrm{L} / \mathrm{min}$ (A: Water $+0.05 \%$ of Formic Acid, B: Acetonitrile, A/B: 30\%/70\%). $40 \mu \mathrm{L}$ of sample injected Column Oven $30^{\circ} \mathrm{C}$, Desolvatation Line $300^{\circ} \mathrm{C}$, Heat $500^{\circ} \mathrm{C}$, Nebulizing Gas $3 \mathrm{~L} / \mathrm{min}$, Drying Gas $20 \mathrm{~L} / \mathrm{min}$. All data collected were reprocessed using Shimadzu Neonatal Software, which automatically calculated the concentration of each compounds.

2.6. Statistical Analysis: All the data analyses were performed using SPSS 20 for Windows software (SPSS Inc., NY, USA). The data were statistically analyzed with the Levene test and Shapiro Wilk test 
for equality of variances and the normality assumption, respectively. Then, data $(\mathrm{C} 0, \mathrm{C} 2, \mathrm{C} 3, \mathrm{C} 4$, C4DC, C5, C5:1, C5OH, C5DC, C6, C6DC, C8, C8:1, C8DC, C10, C10:1, C10DC, C12, C14, C14:1, C14:2, $\mathrm{C} 16, \mathrm{C} 16: 1, \mathrm{C} 18, \mathrm{C} 18: 1, \mathrm{C} 18: 2, \mathrm{C} 18: \mathrm{OH})$ were analyzed using the Mann-Whitney U test to determine any further differences between the groups. Data were presented as mean with standard deviation values. A value of $\mathrm{p}<0.05$ was accepted as statistically significant for all tests.

\section{Results}

\subsection{Subsection}

No significant difference was determined between the LP patient group and the control group in respect of demographic data (Table 1). The results of the free carnitine and acylcarnitine amounts determined with LC-MS/MS in the serum samples of the LP patients (n:14) and the healthy control group (n:14) are presented in Table 2. An increase in C5 and C5:OH was observed in the LP group compared to the control group and a statistically significant increase was determined in C2 and C14 ( $\mathrm{p}<0.05)$. Of the other acylcartinines, C5:1, C5OH, C6DC, C8:1, C16:1, C18:1 and C18:2 were reduced in the LP patients compared to the control group and CO (free carnitine) C3, C4, C4:DC, C5DC, C6, C8, C14:1, C14:2, C16 and C18 were determined to be significantly reduced ( $\mathrm{p}<0.05, \mathrm{p}<0.01$, respectively) (Table 1). The $\mathrm{AC} / \mathrm{FC}$ ratios, as a marker of mitochondrial dysfunction, were compared. The $\mathrm{C} 2 / \mathrm{C} 0, \mathrm{C} 5 \mathrm{OH} / \mathrm{C} 0, \mathrm{C} 5 \mathrm{DC} / \mathrm{C} 0, \mathrm{C} 6 / \mathrm{C} 0, \mathrm{C} 10 / \mathrm{C} 0, \mathrm{C} 10: 1 / \mathrm{C} 0, \mathrm{C} 12 / \mathrm{C} 0, \mathrm{C} 14: 1 / \mathrm{C} 0$, and $\mathrm{C} 14: 2 / \mathrm{C} 0$ ratios were found to be statistically significantly low in the LP patients compared to the control group $(\mathrm{p}<0.05, \mathrm{p}<0.01$ respectively) (Table 3 ).

Table1: The demographic variables of the patient and control groups. The $\mathrm{p}$ values for the comparison of the variables between two groups were calculated according to the Mann-Whitney U test

\begin{tabular}{lcccc}
\hline & Group & $\mathbf{N}$ & Mean \pm Sd & p-value \\
\hline Age (years) & Patient & 14 & $15.07 \pm 7.20$ & 0.927 \\
& Control & 14 & $15.42 \pm 8.54$ & \\
Height (cm) & Patient & 14 & $150.07 \pm 29.82$ & 0.346 \\
& Control & 14 & $144.07 \pm 30.77$ & \\
Body weight (kg) & Patient & 14 & $51.35 \pm 19.70$ & 0.260 \\
& Control & 14 & $46.0 \pm 17.95$ & \\
BMI & Patient & 14 & $21.63 \pm 1.70$ & 0.232 \\
& Control & 14 & $21.13 \pm 1.28$ & \\
\hline
\end{tabular}

Table 2: The acylcarnitines in the serum samples of the two studied groups quantified using the LC-MS/MS method. ${ }^{*} \mathrm{p}<0.05$ and ${ }^{* *} \mathrm{p}<0.001$ values for the comparison of the variables between two groups were calculated according to the Mann-Whitney $\mathrm{U}$ test

\begin{tabular}{cccc}
\hline Acylcarnitines & $\begin{array}{c}\text { Patient (n:14) } \\
\text { mean } \pm \text { sd }\end{array}$ & $\begin{array}{c}\text { Control (n:14) } \\
\text { mean } \pm \text { sd }\end{array}$ & p value \\
\hline C0 & $98.3 \pm 21.77$ & $120.87 \pm 28.72$ & $0.05^{*}$ \\
C2 & $33.57 \pm 9.06$ & $57.17 \pm 9.72$ & $0.001^{*}$ \\
C3 & $0.93 \pm 0.3$ & $1.25 \pm 0.49$ & $0.05^{*}$ \\
C4 & $0.4 \pm 0.21$ & $0.43 \pm 0.25$ & 0.945 \\
C4DC & $0.06 \pm 0.02$ & $0.09 \pm 0.02$ & $0.003^{*}$ \\
C5 & $0.23 \pm 0.1$ & $0.21 \pm 0.08$ & 0.645 \\
C5:1 & $0.18 \pm 0.06$ & $0.22 \pm 0.11$ & 0.475
\end{tabular}




\begin{tabular}{cccc}
$\mathrm{C} 5 \mathrm{OH}$ & $0.16 \pm 0.14$ & $0.11 \pm 0.05$ & 0.145 \\
$\mathrm{C} 5 \mathrm{DC}$ & $0.24 \pm 0.1$ & $0.51 \pm 0.11$ & $0.001^{*}$ \\
$\mathrm{C} 6$ & $0.06 \pm 0.02$ & $0.11 \pm 0.03$ & $0.001^{*}$ \\
$\mathrm{C} 6 \mathrm{DC}$ & $0.04 \pm 0.01$ & $0.04 \pm 0.01$ & 0.698 \\
$\mathrm{C} 8$ & $0.07 \pm 0.09$ & $0.31 \pm 0.21$ & $0.001^{*}$ \\
$\mathrm{C} 8: 1$ & $0.12 \pm 0.06$ & $0.12 \pm 0.07$ & 0.695 \\
$\mathrm{C} 8 \mathrm{DC}$ & $0.03 \pm 0.01$ & $0.05 \pm 0.04$ & $0.037^{*}$ \\
$\mathrm{C} 10$ & $0.21 \pm 0.14$ & $0.75 \pm 0.33$ & $0.001^{*}$ \\
$\mathrm{C} 10: 1$ & $0.39 \pm 0.20$ & $0.98 \pm 0.38$ & $0.001^{*}$ \\
$\mathrm{C} 10 \mathrm{DC}$ & $0.019 \pm 0.01$ & $0.02 \pm 0.001$ & $0.015^{*}$ \\
$\mathrm{C} 12$ & $0.1 \pm 0.05$ & $0.029 \pm 0.01$ & $0.001^{*}$ \\
$\mathrm{C} 14$ & $0.04 \pm 0.02$ & $0.07 \pm 0.02$ & $0.001^{*}$ \\
$\mathrm{C} 14: 1$ & $0.1 \pm 0.06$ & $0.27 \pm 0.12$ & $0.001^{*}$ \\
$\mathrm{C} 14: 2$ & $0.26 \pm 0.15$ & $0.71 \pm 0.3$ & $0.001^{*}$ \\
$\mathrm{C} 16$ & $0.19 \pm 0.05$ & $0.21 \pm 0.05$ & 0.333 \\
$\mathrm{C} 16: 1$ & $0.09 \pm 0.04$ & $0.16 \pm 0.05$ & $0.001^{*}$ \\
$\mathrm{C} 18$ & $0.07 \pm 0.02$ & $0.07 \pm 0.01$ & 0.814 \\
$\mathrm{C} 18: 1$ & $0.04 \pm 0.01$ & $0.05 \pm 0.01$ & $0.014^{*}$ \\
$\mathrm{C} 18: 2$ & $0.1 \pm 0.04$ & $0.11 \pm 0.03$ & 0.105 \\
$\mathrm{C} 18: 1 \mathrm{OH}$ & $0.008 \pm 0.005$ & $0.011 \pm 0.003$ & 0.109 \\
\hline
\end{tabular}

Table3: The AC/FC ratios, as a marker of mitochondrial dysfunction, were compared.

\begin{tabular}{lccc}
\hline $\begin{array}{c}\text { AC/FC } \\
\text { ratios }\end{array}$ & Patient (n:14) & Control (n:14) & p value \\
\hline $\mathrm{C} 2 / \mathrm{C} 0$ & $0.349 \pm 0.1$ & $0.48 \pm 0.07$ & $0.003^{*}$ \\
$\mathrm{C} 3 / \mathrm{C} 0$ & $0.009 \pm 0.002$ & $0.01 \pm 0.003$ & 0.581 \\
$\mathrm{C} 4 / \mathrm{C} 0$ & $0.004 \pm 0.001$ & $0.003 \pm 0.002$ & 0.383 \\
$\mathrm{C} 4 \mathrm{DC} / \mathrm{C} 0$ & $0.0007 \pm 0.0002$ & $0.0007 \pm 0.0002$ & 0.613 \\
$\mathrm{C} 5 / \mathrm{C} 0$ & $0.002 \pm 0.0009$ & $0.001 \pm 0.0004$ & 0.108 \\
$\mathrm{C} 5: 1 / \mathrm{C} 0$ & $0.001 \pm 0.0004$ & $0.001 \pm 0.0007$ & 0.783 \\
$\mathrm{C} 5 \mathrm{OH} / \mathrm{C} 0$ & $0.0016 \pm 0.0009$ & $0.001 \pm 0.0006$ & $0.004^{*}$ \\
$\mathrm{C} 5 \mathrm{DC} / \mathrm{C} 0$ & $0.0024 \pm 0.0009$ & $0.0045 \pm 0.001$ & $0.001^{* *}$ \\
$\mathrm{C} 6 / \mathrm{C} 0$ & $0.0006 \pm 0.0002$ & $0.0009 \pm 0.00034$ & $0.035^{*}$ \\
$\mathrm{C} 6 \mathrm{DC} / \mathrm{C} 0$ & $0.0004 \pm 0.00007$ & $0.0003 \pm 0.0001$ & 0.073 \\
$\mathrm{C} 8 / \mathrm{C} 0$ & $0.0016 \pm 0.0009$ & $0.003 \pm 0.002$ & 0.104 \\
$\mathrm{C} 8: 1 / \mathrm{C} 0$ & $0.0014 \pm 0.0008$ & $0.001 \pm 0.0005$ & 0.383 \\
$\mathrm{C} 8 \mathrm{DC} / \mathrm{C} 0$ & $0.0003 \pm 0.0001$ & $0.0004 \pm 0.0003$ & 0.491 \\
$\mathrm{C} 10 / \mathrm{C} 0$ & $0.0022 \pm 0.0014$ & $0.006 \pm 0.003$ & $0.001^{* *}$ \\
$\mathrm{C} 10: 1 / \mathrm{C} 0$ & $0.0041 \pm 0.0022$ & $0.0085 \pm 0.003$ & $0.001^{* *}$ \\
$\mathrm{C} 10 \mathrm{DC} / \mathrm{C} 0$ & $0.0002 \pm 0.00009$ & $0.0002 \pm 0.00007$ & 0.550 \\
$\mathrm{C} 12 / \mathrm{C} 0$ & $0.0011 \pm 0.00057$ & $0.0026 \pm 0.0011$ & $0.001^{* *}$ \\
$\mathrm{C} 14 / \mathrm{C} 0$ & $0.0004 \pm 0.0002$ & $0.0006 \pm 0.0002$ & 0.027 \\
$\mathrm{C} 14: 1 / \mathrm{C} 0$ & $0.0011 \pm 0.0007$ & $0.0025 \pm 0.0014$ & $0.002^{*}$ \\
$\mathrm{C} 14: 2 / \mathrm{C} 0$ & $0.0028 \pm 0.0017$ & $0.0062 \pm 0.002$ & $0.002^{*}$
\end{tabular}




\begin{tabular}{lccc}
$\mathrm{C} 16 / \mathrm{C} 0$ & $0.0021 \pm 0.001$ & $0.0018 \pm 0.0005$ & 0.462 \\
$\mathrm{C} 16: 1 / \mathrm{C} 0$ & $0.0010 \pm 0.0006$ & $0.0014 \pm 0.0005$ & 0.081 \\
$\mathrm{C} 18 / \mathrm{C} 0$ & $0.0008 \pm 0.00024$ & $0.0006 \pm 0.0001$ & 0.066 \\
$\mathrm{C} 18: 1 / \mathrm{C} 0$ & $0.0005 \pm 0.00026$ & $0.0005 \pm 0.0001$ & 0.291 \\
$\mathrm{C} 18: 2 / \mathrm{C} 0$ & $0.0011 \pm 0.00071$ & $0.001 \pm 0.0002$ & 0.963 \\
$\mathrm{C} 18: 1 \mathrm{OH} / \mathrm{C} 0$ & $0.0001 \pm 0.00005$ & $0.0001 \pm 0.00005$ & 0.298 \\
\hline
\end{tabular}

\section{Discussion}

LP is a chronic autosomal recessive transfer disease characterised by hyalin substance accumulation in the mucous membranes and internal organs. A genetic disorder causes a mutation in the extracellular matrix protein-1 gene and this results in hyalin accumulation [11,12]. LP has a stable or slowly progressive course [13]. When there is no airway obstruction or the occurrence of life-threatening epileptic seizures, this disease is consistent with a normal life course. Mortality generally occurs because of laryngeal obstruction. As the incidence is low and the etiology is associated with a genetic mutation, there is no recommended treatment strategy for intervention on systemic effects that is effective on the prognosis of LP disease. The determination of the metabolic profile of systemic diseases has been useful for biochemical clues of the accumulated metabolites acquired in literature about the disease [13]. With metabolomic and proteomic studies, the determination of new biochemical markers with LC-MS/MS devices, which are often used in congenital metabolic diseases, can contribute to the development of protective and therapeutic strategies [14]. To the best of our knowledge, this is the first study to have examined with LC-MS/MS the levels of free carnitine and acylcarnitine that play a significant role in the transport of fatty acids to the mitochondria in LP, in which there is lipid accumulation in lysosomes.

L-carnitine is an amino acid-like molecule that plays a significant role in the transport of long-chain fatty acids along the internal mitochondrial membrane [15]. Acylcarnitines, which are the esterised form, are reaction products which are transferred from primary CoA of the main parts to carnitine. These main groups vary in several lengths from short chain (acetyl) to long chain (palmitoil).

Carnitine can be synthesised endogenously [16]. The main sites of L-carnitine synthesis are the kidneys, liver and brain. The theory that systemic involvement in LP disease, especially brain involvement, can lead to defects in carnitine metabolism explains the low levels of acylcarnitine that were observed in the patient group [16].

In a study by Hamada et al, the impairment that plays a role in LP pathogenesis was shown to originate from the long arm of chromosome-1 [3]. Chromosome-1 has been related to collagen tissue disease and lipid metabolism disorders [17]. G. Finocchiaro et al demonstrated that there was a relationship between carnitine palmitoiltransferase (CPTase) activity and chromosome 1q12. More than 52 mutations have been identified in LP together with ECM1 that encodes extracellular matrix protein in chromosome 1q12 [2,18]. In histological studies, the disease is characterised by resistance to diastase in the dermoepidermal component, thickening of the basal membrane and accumulation of hyalin material in blood vessels and adnexial epithelium in addition to the dermis. Abnormal lysosomes similar to Farber disease have been shown in LP patients, which are thought to reflect the abnormality in the impaired pathway of glycolipids or sphingolipids. In some studies, LP pathology has been evaluated using biochemical analysis of skin and cultured fibroblasts $[19,20]$. In particular, Bauer et al suggested that because of the complexity of lysosomal material accumulation, there could be multiple enzyme defects together with mucopolysaccharide lipid and glycolipid metabolism disorders [21].

As there is lipid accumulation in this disease, blood acylcarnitine levels are also affected. Carnitines are known to have a significant role in the transport of active fatty acids along the internal mitochondrial membrane and are necessary for energy production through fatty acid metabolism. In addition, carnitines have a place in the removal of accumulated toxic fatty acyl-CoA metabolites and assist in the protection of the balance between free and acyl-CoA. Carnitines are found in the forms 
of free carnitine (non-esterised molecule; FC) or acylcarnitine (esterised form; AC). The AC/FC ratio is a measurement of carnitines that have acquired acyl compared to free carnitines. L-carnitine deficiency that results in functional carnitine deficiency can increase the AC/FC ratio. Other factors such as enzymatic changes in carnitine metabolism can cause higher acylcarnitine levels and therefore, a high AC/FC ratio. Thus, while a low AC/FC ratio shows healthy mitochondria, a high ratio indicates reduced mitochondrial capacity for energy production [22].

As LP cases are rarely seen and few patients can reach advanced diagnostic centres, there are insufficient laboratory data which could provide ideas about the etiopathogenesis of the disease and prognosis. In the current study, C0 (free carnitine) C3, C4, C4:DC, C5DC, C6, C8, C14:1, C14:2, C16 and $\mathrm{C} 18$ acylcarnitine levels, which were considered to be potential biomarkers in LP patients, were determined to be significantly low compared to the values in healthy individuals. The reason for the lower levels determined in the patient group can most probably be associated with genetic defect combined with accumulated metabolites and carnitine metabolism. This change in carnitine metabolism is explained by the multiple enzyme defect theory in LP, as proposed by Bauer et al. However, the determination of lower $\mathrm{C} 2 / \mathrm{C} 0, \mathrm{C} 5 \mathrm{OH} / \mathrm{C} 0, \mathrm{C} 5 \mathrm{DC} / \mathrm{C} 0, \mathrm{C} 6 / \mathrm{C} 0, \mathrm{C} 10 / \mathrm{C} 0, \mathrm{C} 10: 1 / \mathrm{C} 0, \mathrm{C} 12 / \mathrm{C} 0$, $\mathrm{C} 14: 1 / \mathrm{C} 0, \mathrm{C} 14: 2 / \mathrm{C} 0$ ratios in the LP group compared to the healthy control group suggests that mitochondrial capacity was not reduced. Although there was no reduction in the mitochondrial capacity, the reduction in blood concentrations of acylcarnitines in LP, which is a lysosomal metabolic storage disease, necessitates advanced genetic analyses of enzyme defects.

\section{Conclusions}

In this study, which aimed to reveal differences of acylcarnitine levels between LP patients and healthy individuals, LC-MS/MS based metabolomics was successfully applied. The application used in this study of inexpensive, rapid, and reliable carnitine profile screening from dry blood can be considered to provide a contribution to the differential diagnosis as a supportive laboratory test in individuals with suspected LP. With the results obtained in this study, it can be predicted that with the identification of carnitine metabolic pathways, the pathogenesis of LP could be clarified and new treatment protocols could be investigated with interventions to these pathways.

Author Contributions: Conceptualization and writing the original draft, Ataman Gönel; data curation and investigation, Hakim Çelik, Mustafa Aksoy; methodology, supervision and writing review \& editing, Ismail Koyuncu

Funding: This research received no specific grant from any funding agency.

Conflicts of Interest: The authors declare no conflict of interest

\section{References}

1. McGrath, J.A. Lipoid proteinosis. In Handbook of clinical neurology, Elsevier: 2015; Vol. 132, pp 317-322.

2. Youssefian, L.; Vahidnezhad, H.; Daneshpazhooh, M.; Abdollahzadeh, S.; Talari, H.; Khoshnevisan, A.; Chams-Davatchi, C.; Mobasher, R.; Li, Q.; Uitto, J. Lipoid proteinosis: Phenotypic heterogeneity in iranian families with c. 507delt mutation in ecm1. Experimental dermatology 2015, 24, 220-222.

3. Hamada, T.; McLean, W.I.; Ramsay, M.; Ashton, G.H.; Nanda, A.; Jenkins, T.; Edelstein, I.; South, A.P.; Bleck, O.; Wessagowit, V. Lipoid proteinosis maps to 1q21 and is caused by mutations in the extracellular matrix protein 1 gene (ecm1). Human molecular genetics 2002, 11, 833-840.

4. de Rezende Pinto, V.; Bocca, W.; Sgobbi de Souza, P.V.; Pedroso, J.L.; Barsottini, O.G.P. Urbach-wiethe disease presenting with partial seizures, skin lesions and typical neuroimaging features. Clinical neurology and neurosurgery 2014.

5. Gruber, F.; Manestar, D.; Stasic, A.; Grgurevic, Z. Treatment of lipoid proteinosis with etretinate. Acta dermato-venereologica 1996, 76, 154. 
6. Ikegami, M.; Dhami, R.; Schuchman, E.H. Alveolar lipoproteinosis in an acid sphingomyelinase-deficient mouse model of niemann-pick disease. American Journal of Physiology-Lung Cellular and Molecular Physiology 2003, 284, L518-L525.

7. Liu, Y.; Yan, S.; Ji, C.; Dai, W.; Hu, W.; Zhang, W.; Mei, C. Metabolomic changes and protective effect of 1-carnitine in rat kidney ischemia/reperfusion injury. Kidney and Blood Pressure Research 2012, 35, 373-381.

8. Patti, G.J.; Yanes, O.; Siuzdak, G. Innovation: Metabolomics: The apogee of the omics trilogy. Nature reviews Molecular cell biology 2012, 13, 263.

9. Azzari, C.; La Marca, G.; Resti, M. Neonatal screening for severe combined immunodeficiency caused by an adenosine deaminase defect: A reliable and inexpensive method using tandem mass spectrometry. Journal of Allergy and Clinical Immunology 2011, 127, 1394-1399.

10. la Marca, G.; Malvagia, S.; Pasquini, E.; Innocenti, M.; Fernandez, M.R.; Donati, M.A.; Zammarchi, E. The inclusion of succinylacetone as marker for tyrosinemia type $\mathrm{i}$ in expanded newborn screening programs. Rapid Communications in Mass Spectrometry 2008, 22, 812-818.

11. Xu, W.; Wang, L.; Zhang, L.; Han, D.; Zhang, L. Otolaryngological manifestations and genetic characteristics of lipoid proteinosis. The Annals of Otology, Rhinology \& Laryngology 2010, 119, 767.

12. Hougenhouck-Tulleken, V.; Chan, I.; Hamada, T.; Thornton, H.; Jenkins, T.; McLean, W.; McGrath, J.; Ramsay, M. Clinical and molecular characterization of lipoid proteinosis in namaqualand, south africa. British Journal of Dermatology 2004, 151, 413-423.

13. Hamie, L.; Knio, Z.; Abbas, O.; Akel, R.; Bardawil, T.; Kibbi, A.; Kurban, M. Clinical clues early in the lives of individuals with lipoid proteinosis can determine the course of the disease. Clinical and experimental dermatology 2017, 42, 428-430.

14. Rienstra, M.; McManus, D.D.; Benjamin, E.J. Novel risk factors for atrial fibrillation: Useful for risk prediction and clinical decision making? Circulation 2012, 125, e941-e946.

15. Bremer, J. Carnitine--metabolism and functions. Physiological reviews 1983, 63, 1420-1480.

16. Sahajwalla, C.G.; Helton, E.D.; Purich, E.D.; Hoppel, C.L.; Cabana, B.E. Multiple-dose pharmacokinetics and bioequivalence of 1-carnitine 330-mg tablet versus 1-g chewable tablet versus enteral solution in healthy adult male volunteers. Journal of pharmaceutical sciences 1995, 84, 627-633.

17. Schutte, B.; Carpten, J.; Forus, A.; Gregory, S.; Horii, A.; White, P. Report of the sixth international workshop on human chromosome 1 mapping 2000. Cytogenetic and Genome Research 2001, 92, 23-48.

18. Chan, I.; Liu, L.; Hamada, T.; Sethuraman, G.; McGrath, J.A. The molecular basis of lipoid proteinosis: Mutations in extracellular matrix protein 1. Experimental dermatology 2007, 16, 881-890.

19. Moy, L.S.; Moy, R.L.; Matsuoka, L.Y.; Ohta, A.; Uitto, J. Lipoid proteinosis: Ultrastructural and biochemical studies. Journal of the American Academy of Dermatology 1987, 16, 1193-1201.

20. Fleischmajer, R.; Krieg, T.; Dziadek, M.; Altchek, D.; Timpl, R. Ultrastructure and composition of connective tissue in hyalinosis cutis et mucosae skin. Journal of investigative dermatology 1984, 82.

21. Bauer, E.A.; Santa-Cruz, D.J.; Eisen, A.Z. Lipoid proteinosis: In vivo and in vitro evidence for a lysosomal storage disease. Journal of Investigative Dermatology 1981, 76.

22. Sharma, S.; Black, S.M. Carnitine homeostasis, mitochondrial function and cardiovascular disease. Drug Discovery Today: Disease Mechanisms 2009, 6, e31-e39. 